\title{
Erratum zu: Mit Daten und \\ Künstlicher Intelligenz zu digitalen \\ Geschäftsmodellen - Praxisbeispiele \\ aus Produktion, Logistik und \\ Gesundheitswirtschaft
}

Johannes Winter

Erratum zu:

M. Bruhn und K. Hadwich (Hrsg.), Künstliche Intelligenz im

Dienstleistungsmanagement, Forum Dienstleistungsmanagement, https://doi.org/10.1007/978-3-658-34324-8_3

In der Online-Version dieses Kapitels wurde die Zusammenfassung korrigiert.

Die korrigierte Zusammenfassung lautet:

Während die Potenziale und Herausforderungen der digitalen Transformation der Wirtschaft hinlänglich bekannt und gut beforscht sind, mangelt es an konkreten Erfolgsund Umsetzungsbeispielen für daten- und KI-basierte Geschäftsmodellinnovationen. Der Beitrag zeigt, wie über den Einsatz von Künstlicher Intelligenz und Kollaboration in Wertschöpfungsnetzwerken die Datenmonetarisierung in Produktion, Logistik und Gesundheitswirtschaft ermöglicht wird. 